\title{
A Planar Acoustic Field Reconstruction Method Based on Fast Wave Superposition Spectrum and Sparse Sampling
}

\author{
Yang Zhang, ${ }^{1,2}$ Yu-Jiang Wang $\left(\mathbb{D},{ }^{1,2}\right.$ Yu Xiang $\mathbb{D}^{1},{ }^{1}$ Zi-Yu Shi, ${ }^{1,2}$ Shao-Jie Fan, ${ }^{1,2}$ and Jing Lu $\mathbb{D}^{1,2}$ \\ ${ }^{1}$ Key Laboratory of Automobile Component and Vehicle Technology in Guangxi, Guangxi University of Science and Technology, \\ Liuzhou 545006, China \\ ${ }^{2}$ School of Mechanical and Automotive Engineering, Guangxi University of Science and Technology, Liuzhou 545006, China
}

Correspondence should be addressed to Yu Xiang; xiangyu@gxust.edu.cn

Received 23 October 2021; Accepted 7 January 2022; Published 24 January 2022

Academic Editor: Erkan Oterkus

Copyright (c) 2022 Yang Zhang et al. This is an open access article distributed under the Creative Commons Attribution License, which permits unrestricted use, distribution, and reproduction in any medium, provided the original work is properly cited.

Based on the fast Fourier wave superposition spectrum method, a new equivalent source method (ESM) with a sparse sampling technique is proposed. First, the equivalent source intensities are expanded on a rectangular virtual surface using a bidirectional Fourier series, resulting in a semi-analytic and half-numerical acoustic pressure expression. The Fourier coefficients result in good sparsity for continuous acoustic pressures from structural vibration sources, and the proposed sparse sampling method can further reduce correlation in the measurement matrix. Better results can be obtained by solving the $l_{1}$ norm optimization problem. Finally, the method was verified using several examples. The proposed method offers two main advantages compared with the traditional compressive equivalent source method: (1) the unknown source intensity vector is expanded into a bidirectional Fourier series, thereby transforming an unknown source intensity vector into a sparse Fourier coefficient vector, which has better sparsity; (2) the proposed method constructs a random sampling matrix, which is expanded into a sparse sampling matrix by random distribution, thereby improving the reconstruction accuracy of planar near-field acoustic field compared with the traditional random position sampling method reducing correlation in the transfer matrix.

\section{Introduction}

Near-field acoustic holography (NAH) is a powerful technique for identifying and localizing acoustic sources and visualizing acoustic fields [1]. With the development of NAH technology, several new algorithms have been introduced including the spatial Fourier transform (SFT) [2], boundary element method (BEM) [3], and equivalent source method (ESM) [4]. Among them, the ESM has been widely studied and applied, mainly owing to its simple principle and strong adaptability [5]. According to the principle of the ESM, the acoustic field radiated by a vibrating body of arbitrary shape can be approximated as the superposition of the acoustic field generated by a series of virtual equivalent sources distributed inside the structure. The acoustic field is reconstructed using the weight coefficients of each equivalent source based on measured acoustic pressure on a holographic surface [6]. Since noise in measured data cannot be avoided, the least-squares method based on the $l_{2}$ norm is usually used with the traditional ESM to obtain a stable solution. In practical applications of $\mathrm{NAH}$, due to the measurement conditions and cost limitations, the acoustic reconstruction problem usually involves solving a set of underdetermined equations. The least-squares method based on the $l_{2}$ norm is used to solve the undetermined system of equations, which makes it difficult to obtain an ideal reconstruction result.

In recent years, compressed sensing (CS) has been widely used in signal and image processing [7] and has become a hot topic of research $[8,9]$. The novel compressive sampling technique uses the sparsity of the signal to solve the underdetermined system of equations. The sparse solution is easier to obtain because the reconstruction algorithm is based on the $l_{0}$ or $l_{1}$ norm instead of the traditional leastsquares method based on the $l_{2}$ norm. Thus, good reconstruction accuracy is guaranteed with fewer measurement 
points. In 2012, Chardon et al. [10] introduced CS into NAH for the first time and experimentally demonstrated higher accuracy compared with the traditional method when reducing the measurement points. Fernandez and Xenaki [11] proposed the compressive equivalent source method (CESM) by combining CS with ESM and analyzed the influence of column correlation in the transfer matrix on the reconstruction result [12]. On the basis of the C-ESM, Hu et al. [13] studied the reconstruction of sparse sound field through the sparse basis obtained by singular value decomposition of the transfer matrix; a fast sparse acoustic field reconstruction method was proposed to combine the Bayesian compressed sensing with the sparse basis function in the following study [14]. In addition to the C-ESM, there are many methods combining compressed sensing with equivalent source method, for example, compressed fused model equivalent source method (CFMESM) [15], compressed velocity-mode equivalent source method (CVMESM) [16], and fused total generalized variation (FTGV) [17]. The particle velocity maps will have sharp peaks in CVMESM [15]. The mode scaling factor was set in CFMESM, and the need for the parameter is a weakness of the method [16]. FTGV combines sparsity in the second derivatives with sparsity in the amplitudes, but FTGV must be solved using the CVX toolbox [17]. In previous studies, sampling points (microphones) are often randomly arranged to satisfy noncorrelation between the observation matrix and the sparse basis. Random sampling can reduce correlation in the measurement matrix through random positioning. Moreover, since previous methods are based on the assumed sparsity of the equivalent source intensities, it is difficult to obtain accurate prior information of the acoustic source when the type of acoustic source is unknown, and the sparsity requirement is not guaranteed. Using corresponding sparse sampling to the transform matrix, we can not only randomly select the sampling position to reduce correlation in the measurement matrix but also use the random coefficient matrix to further reduce correlation.

In this study, a planar acoustic field reconstruction method based on the fast wave superposition spectrum and the sparse sampling matrix is proposed. The bidirectional Fourier series expansion of the equivalent source intensity on a rectangular virtual surface is used to establish a semianalytical and semi-numerical Fourier series expansion form, and the sparsity of the Fourier coefficient vector is analyzed. To reduce the correlation of the measurement matrix further, a sampling matrix with random spatial positioning and random summation coefficients is introduced. Finally, simulations were performed to compare the proposed method and the traditional C-ESM for planar acoustic field reconstruction.

\section{Planar Acoustic Field Reconstruction Based on Fast Fourier Wave Superposition Spectrum}

2.1. Fourier Series Expansion of Acoustic Pressure. From the wave superposition method (WSM) [4], the acoustic pressure at any field point $\mathbf{r}$ outside the acoustic source can be superposed by a series of virtual equivalent sources in the virtual surface $S_{E}$ inside the acoustic source. Thus, the acoustic pressure can be expressed using the following integral:

$$
p(\mathbf{r})=\int_{S_{E}} q\left(\mathbf{r}_{E}\right) G\left(\mathbf{r}, \mathbf{r}_{E}\right) \mathrm{d} S_{E}
$$

where $\mathbf{r}_{E}$ is the position vector of the equivalent source, $q\left(\mathbf{r}_{E}\right)$ is the strength of the equivalent source at $\mathbf{r}$, and $G\left(\mathbf{r}, \mathbf{r}_{E}\right)$ is Green's function. As shown in Figure 1, the position coordinates of field point $\mathbf{r}$ and equivalent source point $\mathbf{r}_{E}$ in the rectangular coordinate system are $\mathbf{r}=(x, y, z)$ and $\mathbf{r}_{E}=\left(x_{E}, y_{E}, z_{E}\right)$, respectively. To reconstruct the planar acoustic field, the virtual surface $S_{E}$ is arranged in a rectangular domain of size $2 L_{x} \times 2 L_{y}$.

Expanding the equivalent source intensity $q\left(\mathbf{r}_{E}\right)$ in (1) into a Fourier series along the $x$-axis and $y$-axis of the selected virtual surface $S_{E}, z_{E}$ is given, and it becomes the following:

$$
q\left(\mathbf{r}_{E}\right)=\sum_{m=-\infty}^{+\infty} \sum_{n=-\infty}^{+\infty} C_{m n}\left(z_{E}\right) \exp \left(-\mathrm{i} \frac{m \pi x_{E}}{L_{x}}\right) \exp \left(-\mathrm{i} \frac{n \pi y_{E}}{L_{y}}\right), \quad x_{E} \in\left[-L_{x}, L_{x}\right], y \in\left[-L_{y}, L_{y}\right]
$$

where the Fourier series coefficient is as follows: $C_{m n}\left(z_{E}\right)=1 / 4 L_{x} L_{y} \quad \iint_{S_{E}} q\left(\mathbf{r}_{E}\right) \exp \left(\mathrm{i} m \pi x_{E} / L_{x}\right) \exp \left(\mathrm{i} n \pi y_{E} /\right.$ $\left.L_{y}\right) \mathrm{d} x_{E} \mathrm{~d} y_{E}$.
Substituting equation (2) into equation (1), the pressure field becomes

$$
p(\mathbf{r})=\sum_{m=-\infty}^{+\infty} \sum_{n=-\infty}^{+\infty} C_{m n}\left(z_{E}\right) \int_{-L_{y}}^{L_{y}} \int_{-L_{x}}^{L_{x}} G\left(\mathbf{r} ; x_{E}, y_{E}, z_{E}\right) \exp \left(-\mathrm{i} \frac{m \pi x_{E}}{L_{x}}\right) \exp \left(-\mathrm{i} \frac{n \pi y_{E}}{L_{y}}\right) \mathrm{d} x_{E} \mathrm{~d} y_{E}
$$




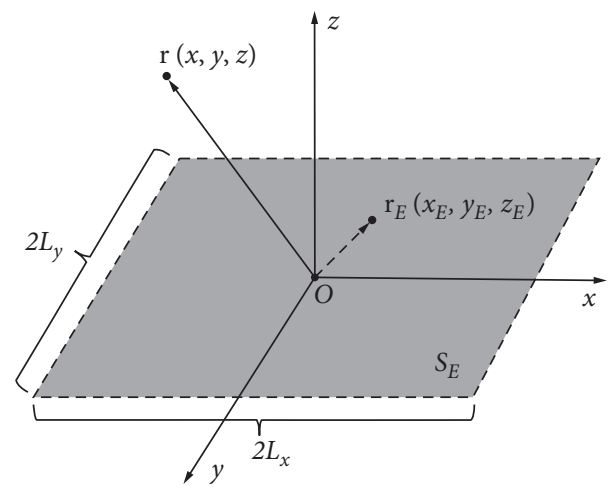

FIgURE 1: Field point and source point.

In practical calculations, the summation range of the series in equation (3) needs to be truncated; that is,

$$
p(\mathbf{r})=\sum_{m=-m_{f}}^{m_{f}} \sum_{n=-n_{f}}^{n_{f}} C_{m n}\left(z_{E}\right) \int_{-L_{y}}^{L_{y}} \int_{-L_{x}}^{L_{x}} G\left(\mathbf{r} ; x_{E}, y_{E}, z_{E}\right) \exp \left(-\mathrm{i} \frac{m \pi x_{E}}{L_{x}}\right) \exp \left(-\mathrm{i} \frac{n \pi y_{E}}{L_{y}}\right) \mathrm{d} x_{E} \mathrm{~d} y_{E} .
$$

$$
\bar{G}_{m n}\left(\mathbf{r} ; z_{E}\right)=\int_{-L_{y}}^{L_{y}} \int_{-L_{x}}^{L_{x}} G\left(\mathbf{r} ; x_{E}, y_{E}, z_{E}\right) \exp \left(-\mathrm{i} \frac{m \pi x_{E}}{L_{x}}\right) \exp \left(-\mathrm{i} \frac{n \pi y_{E}}{L_{y}}\right) \mathrm{d} x_{E} \mathrm{~d} y_{E} .
$$

Dividing the integration range $\left(-L_{x} \sim L_{x}\right)$ and $\left(-L_{y} \sim L_{y}\right)$ in equation (5) into equal divisions of $M$ and $N$, respectively, it becomes

$$
\begin{cases}\Delta x_{E}=\frac{2 L_{x}}{M}, & x_{E}=k_{1} \Delta x_{E}, \quad k_{1}=0,1,2 \ldots M-1, \\ \Delta y_{E}=\frac{2 L_{y}}{N}, & y_{E}=k_{2} \Delta y_{E}, \quad k_{2}=0,1,2 \ldots N-1 .\end{cases}
$$

Using the trapezoidal formula to numerically solve equation (5), it becomes

$$
\begin{aligned}
\bar{G}_{m n}\left(\mathbf{r} ; z_{E}\right) & =\frac{4 L_{x} L_{y}}{M N} \sum_{k_{1}=0}^{M-1} \sum_{k_{2}=0}^{N-1} G\left(\mathbf{r} ; \frac{2 k_{1} L_{x}}{M}, \frac{2 k_{2} L_{y}}{N}, z_{E}\right) \exp \left(-\mathrm{i}\left(\frac{2 \pi}{M}\right) m k_{1}\right) \exp \left(-\mathrm{i}\left(\frac{2 \pi}{N}\right) n k_{2}\right) \\
m & =0,1,2 \ldots M-1 ; n=0,1,2 \ldots N-1 .
\end{aligned}
$$

The two-dimensional discrete Fourier transform (DFT) and inverse DFT are defined as [18] follows: 


$$
\left\{\begin{array}{ll}
X(m, n)=\sum_{k_{1}=0}^{M-1} \sum_{k_{2}=0}^{N-1} x\left(k_{1}, k_{2}\right) \exp \left(-\mathrm{i}\left(\frac{2 \pi}{M}\right) m k_{1}\right) \exp \left(-\mathrm{i}\left(\frac{2 \pi}{N}\right) n k_{2}\right), \quad m=0,1, \ldots, M-1 ; \quad n=0,1, \ldots, N-1 \\
x\left(k_{1}, k_{2}\right)=\frac{1}{M N} \sum_{m=0}^{M-1} \sum_{n=0}^{N-1} X(m, n) \exp \left(\mathrm{i}\left(\frac{2 \pi}{M}\right) m k_{1}\right) \exp \left(\mathrm{i}\left(\frac{2 \pi}{N}\right) n k_{2}\right), \quad k_{1}=0,1, \ldots, M-1 ; \quad k_{2}=0,1, \ldots, N-1
\end{array},\right.
$$

where the value of $M \times N$ samples is as follows: $x\left(k_{1}, k_{2}\right)\left(k_{1}=0,1, \ldots, M-1 ; k_{2}=0,1, \ldots, N-1\right)$.

It can be seen from equation (8) that equation (7) is the standard two-dimensional DFT. When $M$ and $N$ are positive integer powers of $2, \bar{G}_{m n}\left(\mathbf{r} ; z_{E}\right)$ can be quickly calculated using the discrete fast Fourier transform.

It is worth mentioning that when the DFT is used to calculate $\bar{G}_{m n}\left(\mathbf{r} ; z_{E}\right)$, the range of $m$ and $n$ is $m=(0 \sim M-$ 1) and $n=(0 \sim N-1)$, respectively. However, the summation ranges in equation (4) are $m=\left(-m_{f} \sim+m_{f}\right)$ and $n=\left(-n_{f} \sim+n_{f}\right)$, respectively, and only need to be adjusted by the periodicity of $\bar{G}_{m n}\left(\mathbf{r} ; z_{E}\right)$ with respect to $M$ and $N$.

The fast Fourier wave superposition spectrum of acoustic pressure at any point can be obtained using equations (4) and (7):

$$
p(\mathbf{r})=\sum_{m=-m_{f}}^{m_{f}} \sum_{n=-n_{f}}^{n_{f}} C_{m n}\left(z_{E}\right) \bar{G}_{m n}\left(\mathbf{r} ; z_{E}\right) .
$$

The number of summation truncation terms requires $\left(2 m_{f}+1\right) \leq M$ and $\left(2 n_{f}+1\right) \leq N$.

For acoustic field reconstruction in NAH, measured holographic acoustic pressure data are substituted into equation (9) and written in matrix form:

$$
\mathbf{P}_{\mathbf{h}}=\mathrm{KC} \text {, }
$$

where $\mathbf{P}_{\mathbf{h}}$ is the column vector of acoustic pressure on the holographic surface at sampling point $M_{h}$ and $\mathbf{C}$ is the column vector composed of $M_{v}=\left(2 m_{f}+1\right)\left(2 n_{f}+1\right)$ coefficients $C_{m n}\left(z_{E}\right)$ of the Fourier series of equivalent source intensity on the virtual surface $S_{E}$. K is the transfer matrix of $M_{h} \times M_{v}$ between the acoustic pressure on the holographic sampling surface and the Fourier coefficient of the equivalent source intensity on the virtual surface $S_{E}$. Then, the least-squares method based on the $l_{2}$ norm is used to solve equation (10):

$$
\arg \min \left\|\mathbf{P}_{\mathbf{h}}-\mathbf{K C}\right\|_{2}+\lambda\|\mathbf{C}\|_{2}
$$

where $\lambda$ is the regularization parameter and $\|\cdot\|_{2}$ is the $l_{2}$ norm.

After solving the Fourier coefficient column vector $\mathbf{C}$ of equation (10), the acoustic pressure of any reconstructed surface in the acoustic field can be obtained as follows:

$$
\mathbf{P}_{r}=\mathrm{DC} \text {, }
$$

where $\mathbf{D}$ is the transfer matrix between the acoustic pressure on any reconstructed surface and the Fourier coefficient $C_{m n}\left(z_{E}\right)$ of the equivalent source strength on the virtual surface $S_{E}$.
From the above derivation, the equivalent source intensity $q\left(\mathbf{r}_{E}\right)$ on the virtual surface $S_{E}$ can be expanded into a Fourier series and the acoustic pressure expression can be obtained using the fast Fourier wave superposition spectrum. Since this formula is semi-analytical and semi-numerical, the solution accuracy will be higher than that of the traditional ESM [19]. To improve the calculation results, the traditional least-squares method based on the $l_{2}$ norm must satisfy $M_{h} \geq M_{v}$ and equation (10) must be a set of overdetermined equations.

In practical applications of NAH, the number of holographic measuring points is often insufficient to meet the above requirements due to measurement conditions and cost constraints. Therefore, equation (10) is generally a set of underdetermined equations, making it difficult to obtain satisfactory results using the least-squares method based on the $l_{2}$ norm. However, if the sparsity of the Fourier coefficient vector $\mathbf{C}$ of equivalent source intensity is known, sparse sampling can be carried out on the holographic surface and the underdetermined equations can be solved using the $l_{0}$ or $l_{1}$ norm methods of CS, which can obtain a better solution result.

According to the theory of structural dynamics, the dynamic response (vibration velocity) of a structure under an accidental load excitation is mainly composed of a superposition of low-order modes. Then, the Rayleigh integral shows that the radiated acoustic field is also mainly concentrated in the low-order modes. In other words, the contribution of structural vibration to the acoustic field is mainly concentrated in the low-order modes, whereas the high-order modes are similar to the evanescent modes in the SFT. The higher the order, the faster the attenuation; that is, the higher-order $C_{m n}$ becomes smaller and smaller. Therefore, provided that the number of summation truncation terms $m_{f}$ and $n_{f}$ of equation (9) is large enough, the acoustic field represented by this formula will contain both low-order modes with strong radiation ability and evanescent modes. Thus, the Fourier coefficient vector $C_{m n}$ must be a sparse vector with certain sparsity. As the number of summation truncation terms $m_{f}$ and $n_{f}$ increases, the sparsity is strengthened. Therefore, the proposed fast Fourier wave superposition spectrum method can directly solve the acoustic field reconstruction using sparse sampling combined with the $l_{0}$ or $l_{1}$ norm of CS.

To intuitively explain the sparsity of the Fourier coefficient vector $C_{m n}$, a simple analysis of the sparsity of the Fourier coefficient vector $C_{m n}$ of this kind of continuous vibration acoustic source is presented. The simulation calculations were performed with the vibration of a simply supported plate as the acoustic source and compared with 
the results of the C-ESM [12]. The dimensions of the simply supported plate were $0.5 \mathrm{~m} \times 0.5 \mathrm{~m} \times 0.03 \mathrm{~m}$ (length $\times$ width $\times$ height). A harmonic excitation force of $1 \mathrm{~N}$ was applied at the center of the plate, and the excitation point was located at the center of the simply supported plate. The sampled acoustic pressure on the holographic surface was calculated by the Rayleigh integral method to obtain the theoretical acoustic pressure at the measuring point [20]. The Gaussian white noise with a signal-to-noise ratio (SNR) of $20 \mathrm{~dB}$ was added to simulate the actual acoustic pressure. The holographic surface was $0.1 \mathrm{~m}$ above the simply supported plate, the reconstruction surface was $0.05 \mathrm{~m}$ above the simply supported plate, and the virtual equivalent source surface was arranged on the simply supported plate. Sizes of the holographic surface, reconstruction surface, and virtual equivalent source surface are consistent with those of the simply supported plate. In the C-ESM, 81 measuring points (a $9 \times 9$ uniform array) were set on the holographic surface and the number of equivalent sources was 441 . According to the relationship between the number of summation truncation terms and the sound wavelength, the number of summation and truncation terms in the $x$ and $y$ directions was $n_{f}=5$ and $m_{f}=20$, respectively. According to equation (9), the number of integral segments in the $x$ and $y$ directions of the virtual surface was set as $N=M=2^{9}$. The equation was solved by basis pursuit de-noising (BPDN) based on the $l_{1}$ norm in the SPGL1 toolbox in MATLAB [21].

The amplitudes obtained using the C-ESM and the method in this study with a sampling rate of $1000 \mathrm{~Hz}$ are presented in Figure 2. The virtual source intensity $\mathbf{Q}$ obtained by the C-ESM has large amplitudes at most of the source intensity sequence points and the sparsity is poor, as shown in Figure 2(a). The Fourier coefficient vector $C_{m n}$ of this study has only a small number of large amplitudes and the rest are close to zero, as shown in Figure 2(b), which indicates that the Fourier coefficient vector $C_{m n}$ has stronger sparsity for continuous structural vibration sources such as the simply supported plate. The larger the number of truncated terms $m_{f}$ and $n_{f}$, the stronger the sparsity. Therefore, compared with the C-ESM, the vector has better sparsity and can be better solved using the $l_{1}$ norm.

\section{Principle of Compressed Sensing and Sparse Sampling Matrix}

3.1. Principle of Compressed Sensing. Assuming an N-dimensional signal $\mathbf{x} \in R^{N \times 1}$ can be linearly represented by a set of basis vectors $\psi_{i}$ and defining a matrix composed of basis vectors as $\psi=\left[\psi_{1}, \psi_{1}, \psi_{3} \ldots, \psi_{N}\right]$, then the signal $\mathbf{x}$ can be expressed as follows:

$$
\mathbf{x}=\sum_{i=1}^{N} \psi_{i} \boldsymbol{\alpha}_{i}=\boldsymbol{\psi} \boldsymbol{\alpha},
$$

where $\alpha$ is the decomposition coefficient. If there are only $K$ nonzero values in the decomposition coefficient vector $\alpha$ and $K \ll N$, the sparsity of the coefficient vector $\alpha$ is $K$ and $\psi$ is the sparse basis matrix of signal $\mathbf{x}$. The signal $\mathbf{x}$ is sampled $M$ times based on CS through the measurement matrix $\mathbf{G}$, which is not related to the sparse basis matrix, to obtain the measurement value:

$$
\begin{aligned}
\mathbf{y} & =\mathbf{G} \boldsymbol{\psi} \boldsymbol{\alpha} \\
& =\mathbf{A}_{C S} \boldsymbol{\alpha},
\end{aligned}
$$

where $\mathbf{A}_{C S}$ is a matrix of $M \times N$. Since the decomposition coefficient vector $\alpha$ has some sparsity, it can be optimally solved using the $l_{0}$ norm of CS:

$$
\arg \min \left\|\mathbf{y}-\mathbf{A}_{\mathbf{C S}} \boldsymbol{\alpha}\right\|_{2}+\lambda\|\boldsymbol{\alpha}\|_{0} .
$$

However, the solution of equation (15) belongs to the NP-hard problem, which means that the nondeterministic polynomial (NP) cannot be solved by an exact algorithm, and an effective approximation algorithm for such problems must be sought. To obtain the correct solution, it is necessary to exhaustively enumerate $C_{N}^{K}$ combinations, which will consume a lot of calculation time. Usually, the $l_{1}$ norm is used instead of the $l_{0}$ norm to obtain

$$
\arg \min \left\|\mathbf{y}-\mathbf{A}_{\mathbf{C S}} \boldsymbol{\alpha}\right\|_{2}+\lambda\|\boldsymbol{\alpha}\|_{1} \text {. }
$$

3.2. Construction of Sparse Sampling Matrix. In a previous study, the C-ESM reduced correlation in the measurement matrix (transfer matrix) by randomly arranging the sensor positions [12]. In this study, a random sampling matrix is constructed to reduce the correlation. There is a random matrix of $M_{h^{\prime}} \times M_{h}: \Phi=\left[\varphi_{1} \ldots \varphi_{j} \ldots \varphi_{M_{h}}\right]$, where $M_{h}$ is the number of actual sampling points (microphones). Extending this random matrix to a sampling matrix $\mathbf{S}$ of $M_{h^{\prime}} \times M_{v}$ and randomly distributing $M_{h}$ column vectors in $\Phi$ to $\mathbf{S}$ satisfies $M_{h} \ll M_{v}$, that is $\mathbf{S}=\left[0 \cdots 0 \cdots \varphi_{1} \cdots 0 \cdots \varphi_{2} \cdots 0 \cdots 0 \cdots \varphi_{j} \cdots 0 \cdots \varphi_{M_{h}}\right]$.

Zero column vectors of matrix $S$ are the positions of no sampling points. Multiplying both sides of the sampling matrix in equation (10), it becomes

$$
\mathrm{SP}_{\mathrm{h}}=\mathrm{SKC} \text {. }
$$

Let $\bar{P}_{h}=\mathbf{S P}_{h}$ and $\mathbf{H}=\mathbf{S K}$;

$$
\bar{P}_{h}=\mathrm{HC},
$$

where $\bar{P}_{h}$ is the column vector of $M_{h} \times 1$ and $\mathrm{H}$ is the matrix of $M_{h} \times M_{v}$. Equation (18) is a set of underdetermined equations that can be solved using the $l_{1}$ norm minimization method, that is,

$$
\arg \min \left\|\bar{P}_{h}-\mathrm{HC}\right\|_{2}+\lambda\|\mathbf{C}\|_{1} .
$$

In contrast to the random arrangement of sampling points used in the traditional methods [12], this study proposes a new sparse sampling method. A random matrix is constructed and expanded into the sampling matrix. However, the method of selecting the random sampling matrix is important. Commonly used random sampling matrices are as follows:

(1) Random matrix: construct a random matrix of $M_{h^{\prime}} \times M_{h}$, with values of the array elements evenly 


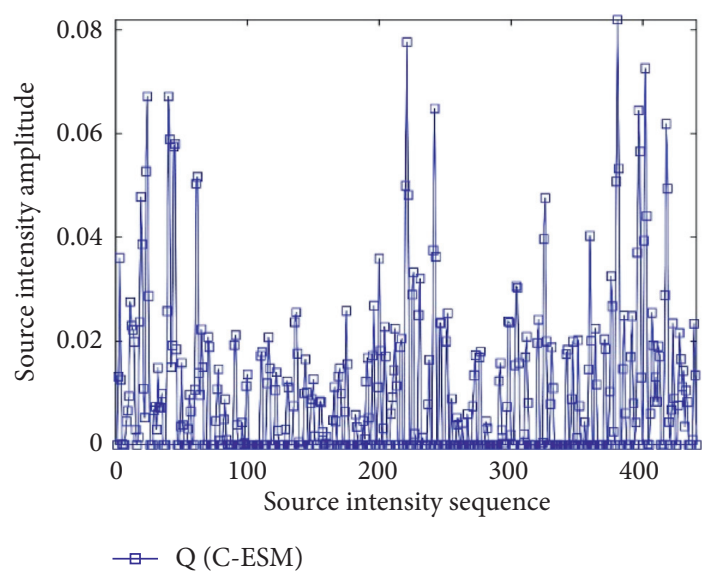

(a)

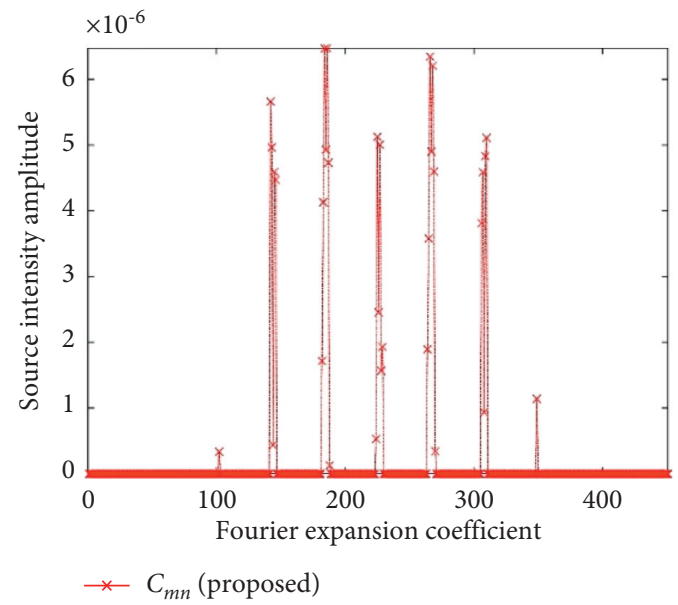

(b)

Figure 2: Amplitude obtained using the compressive equivalent source method and the method with a sampling rate of $1000 \mathrm{~Hz}$. (a) Compressive equivalent source method. (b) Method of this study.

distributed between 0 and 1 . Measurement matrix $\mathrm{P}$ can be obtained by normalizing the column vector.

(2) Gaussian matrix [22]: construct a matrix $\Phi$ of $M_{h^{\prime}} \times M_{h}$ such that each element of the matrix independently satisfies a Gaussian distribution with mean 0 and variance $1 / M_{h^{\prime}}$ (strongly random, not related to most orthogonal bases).

(3) Bernoulli matrix [23]: construct a matrix $\Phi$ of $M_{h^{\prime}} \times M_{h}$ such that every element in the matrix obeys a Bernoulli distribution, independently. Compared with the Gaussian matrix, the elements of the Bernoulli matrix are \pm 1 , which are easier to implement and store in practical applications.

(4) Circulant matrix [24]: first generate an $M_{h}$ dimensional random vector, circulate it $M_{h}\left(M_{h^{\prime}} \leq M_{h}\right)$ times to construct the remaining $M_{h^{\prime}}-1$ row vector, and finally normalize the column vector to obtain the measurement matrix $\Phi$.

(5) Part Fourier matrix [25]: first generate an orthogonal matrix of $M_{h} \times M_{h}$, then randomly select $M_{h^{\prime}}$ row vectors of the orthogonal matrix, and finally normalize the column vectors of $M_{h^{\prime}} \times M_{h}$ to obtain the measurement matrix $\Phi$.

In this study, the above five random sampling matrices are used, and sparse sampling matrices with random distributions are constructed. Compared with the traditional randomly selected sampling positions for reducing correlation in the measurement matrix, the randomly distributed coefficient matrix ensures that the sparse sampling matrix also has the randomness of the summation coefficients of the acoustic pressure at the measurement points. Therefore, the sparse sampling matrix contains not only the randomness of the position but also the randomness of the summation coefficients of the acoustic pressure at measurement points, which further reduces correlation in the sparse sampling matrix.

\section{Simulation Analysis}

4.1. Simply Supported Plate Acoustic Source under Central Excitation. Since the C-ESM has been compared with FTGV, CVMESM, and CFMESM in reference [26], o reduce the amount of computation, the proposed method was only compared with the C-ESM method. To verify the correctness of the proposed method and the accuracy of the acoustic field reconstruction, a simply supported plate was used as a vibration source for simulation calculations. The size of the simply supported steel plate was $0.5 \mathrm{~m} \times 0.5 \mathrm{~m}$, and the thickness was $0.003 \mathrm{~m}$. The plate was driven by a harmonic excitation force of $1 \mathrm{~N}$, and the excitation point was located at the center of the plate. A theoretical radiation acoustic field was calculated using the Rayleigh first integral [20]. The holographic surface was located $0.05 \mathrm{~m}$ above the plate, and its dimensions were consistent with the acoustic source surface. The reconstruction surface was located $0.05 \mathrm{~m}$ from the holographic surface, and the equivalent source surface was arranged on the acoustic source surface.

Dimensions of the reconstruction surface and equivalent source surface were consistent with the holographic surface, and the center of all three surfaces was on the $z$-axis with the center of the plate. In simulations, the actual measured acoustic pressure on the holographic surface was obtained by adding $30 \mathrm{~dB}$ of the Gaussian white noise to the theoretically calculated acoustic pressure. For the C-ESM, the number of virtual equivalent source points was 625 and the number of holographic measurement points was 81 . The sampling points were randomly selected from 625 measurement points on the $25 \times 25$ holographic grid. The distribution of sampling point locations is shown in Figure 3. When the calculations were performed using the method of this study, the microphone array on the measurement surface was consistent with [12]. According to the relationship between the number of summation truncation terms and the sound wavelength, the number along the $x$ and $y$ directions of summation truncation terms was $n_{f}=30$ and 


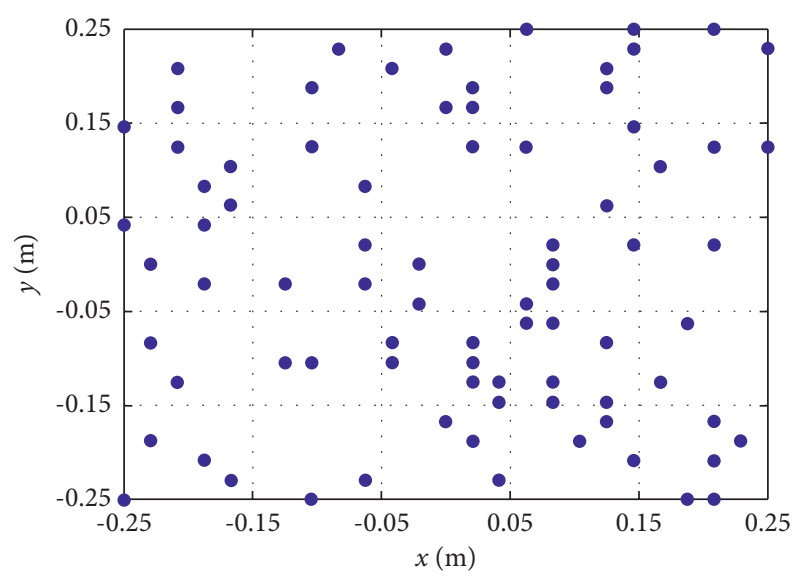

FIgURE 3: Distribution of holographic measurement points for compressive equivalent source method and proposed method.

$m_{f}=40$, respectively. According to equation (9), the sampling number along the $x$ and $y$ directions of the virtual surface was $N_{f}=M_{f}=2^{9}$.

The reconstruction error is defined as follows:

$$
\xi=\frac{\|\mathbf{P}-\overline{\mathbf{P}}\|_{2}}{\|\overline{\mathbf{P}}\|_{2}} \times 100 \%,
$$

where $\mathbf{P}$ is the reconstructed acoustic pressure and $\bar{P}$ is the analytic expression of acoustic pressure.

Figure 4 shows the reconstructed acoustic pressure and theoretical acoustic pressure obtained using the C-ESM or proposed method with five different sparse sampling matrices at frequencies of $500 \mathrm{~Hz}, 1500 \mathrm{~Hz}$, and $2500 \mathrm{~Hz}$. When the frequency is $500 \mathrm{~Hz}$, all reconstructed pressures match the analytical acoustic pressure, except for the proposed method, which combines part of the Fourier matrix. As the frequency increases, the C-ESM can no longer match the analytical acoustic pressure well, whereas the five different sparse sampling matrices are sufficient for obtaining better results with the proposed method. It can also be seen that the proposed method results better reconstruction effects when the Gaussian matrix, Bernoulli matrix, and circular measurement matrix were used as the random sampling measurement matrix and was in better agreement with the analytic expression of acoustic pressure.

Figure 4 is only the reconstruction results at three specific frequencies: $f=500 \mathrm{~Hz}, 1500 \mathrm{~Hz}$, and $2500 \mathrm{~Hz}$. To analyze the reconstruction results at $f=100 \sim 3000 \mathrm{~Hz}$, Figure 5 shows the reconstruction error curves of the C-ESM and the proposed method with five different random sampling measurement matrices in the frequency band from 100 to $3000 \mathrm{~Hz}$. The reconstruction error of the proposed method is lower than that of the C-ESM. Meanwhile, it is difficult to ensure stable reconstruction results in the computational frequency band using either the partial Fourier coefficient matrix or random matrix as the random sampling measurement matrix. The proposed method obtains good results with the Gaussian matrix, Bernoulli matrix, and circular measurement matrix, and the error curves were almost coincident.
4.2. Simple Supported Plate Acoustic Source under Eccentric Excitation. To compare the acoustic field reconstruction of the proposed method and the C-ESM for a simply supported plate acoustic source under eccentric excitation, the parameters of the simply supported plate in Section 3.1 were used. The excitation point was located on the top right of the plate $(0.375,0.375)$, as shown in Figure 6 . The holographic surface was set at $0.06 \mathrm{~m}$ above the plate, and its size was consistent with the acoustic source surface. The reconstruction surface was $0.04 \mathrm{~m}$ above the plate, and the equivalent source surface was $0.01 \mathrm{~m}$ above the acoustic source surface. The sizes of the reconstruction surface and the equivalent source surface were consistent with those of the holographic surface.

In simulations, the same measured acoustic pressure on the holographic surface was used as in the previous example of Section 3.1, which was obtained by adding $30 \mathrm{~dB}$ of the Gaussian white noise to the theoretically calculated acoustic pressure. When the C-ESM is used for calculation, the number of virtual equivalent source points was 484 and the number of holographic measuring points was 64. The number of sampling points was randomly selected from the 484 measuring points on the $22 \times 22$ holographic grid (intervals of $0.024 \mathrm{~m}$ ). The method used for selecting the random sampling matrix in this study was the same as that used in the C-ESM.

Because of the particularity of central excitation, it is more universal to choose eccentric excitation as random excitation. Figure 7 shows the theoretical acoustic pressures of the reconstructed surface at frequencies of $500 \mathrm{~Hz}$, $1500 \mathrm{~Hz}$, and $2000 \mathrm{~Hz}$ and acoustic pressure contours reconstructed using 64 sampling points using either the C-ESM or the proposed method with five different sparse sampling matrices. When $f=500 \mathrm{~Hz}$, the reconstructed acoustic pressures of all methods are in good agreement with the analytical acoustic pressure and the error is less than $10 \%$. As the frequency increases, the C-ESM no longer matches the analytical acoustic pressure well, but the proposed method has high accuracy with five different sparse sampling matrices. Using the Gaussian matrix, Bernoulli matrix, and circular measurement matrix as the random sampling measurement matrix, the proposed method can identify richer details of the acoustic field information.

Figure 8 shows the reconstruction error curves of the C-ESM and the proposed method with five different sparse sampling measurement matrices in the frequency band of $100 \sim 2000 \mathrm{~Hz}$. The reconstruction error of the proposed method is lower than that of the C-ESM. Meanwhile, the reconstruction errors using the partial Fourier coefficient matrix or random matrix are higher than the other three methods; the proposed method obtains good results with the Gaussian matrix, Bernoulli matrix, and circular measurement matrix.

According to Figures 5 and 8, the errors of the C-ESM and the proposed method with five different sparse sampling measurement matrices are less than $20 \%$ in the $100 \sim 1400 \mathrm{~Hz}$ frequency range, and they are all acceptable error percentages. Due to different excitations, the calculation accuracy of five different sparse sampling measurement 


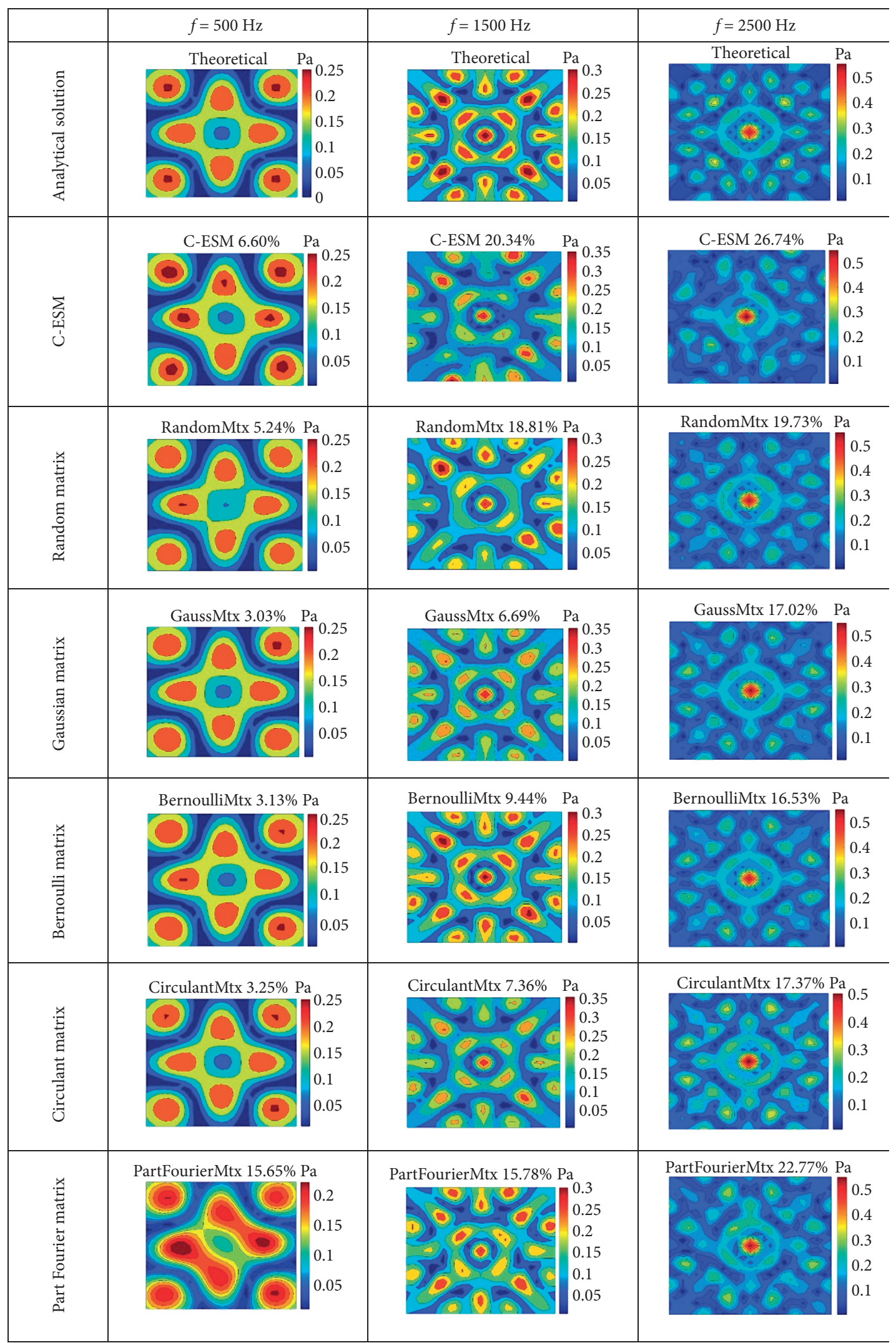

FIgURE 4: Reconstructed and theoretical acoustic pressure nephogram of six methods. 


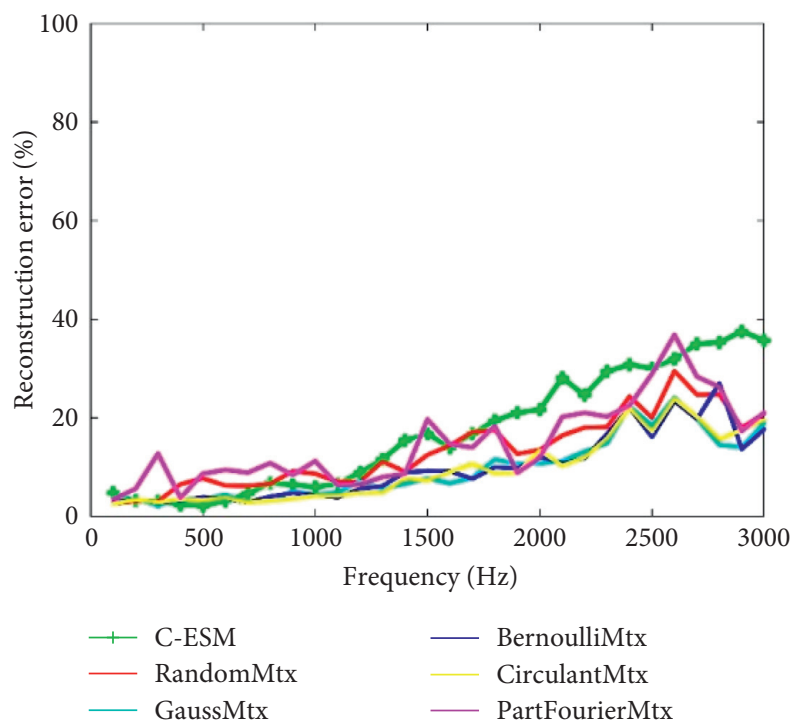

Figure 5: Acoustic pressure reconstruction error with frequency for various methods at 81 sampling points.

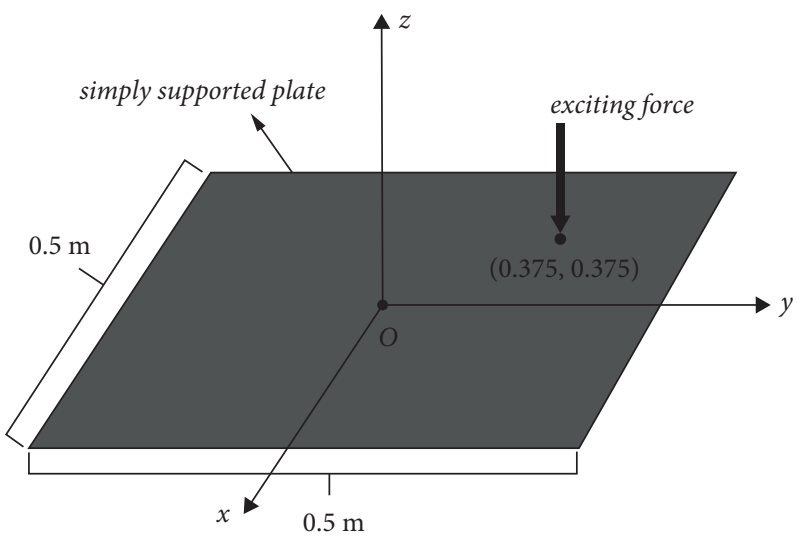

Figure 6: Eccentric excitation point.

matrices is also different, the reconstruction results of the Gaussian matrix, Bernoulli matrix, and circular measurement matrix are better than that of the C-ESM, and the reconstruction results of the partial Fourier coefficient matrix or random matrix are similar to that of the C-ESM. However, the error of the C-ESM under central excitation exceeds $20 \%$, the errors of the Gaussian matrix and circular measurement matrix are about $10 \sim 16 \%$ in the $1400 \sim 3000 \mathrm{~Hz}$ frequency range, and the errors of the Gaussian matrix and circular measurement matrix are acceptable error percentages. Because of the construction characteristic of the Gaussian matrix and circular measurement matrix, satisfactory results could be obtained even in high frequencies. The proposed method with the Gaussian matrix and circular measurement matrix can apply in further studies. In conclusion, the reconstruction results of the Gaussian matrix and circular measurement matrix are the best in five different sparse sampling measurement matrices, and it is 


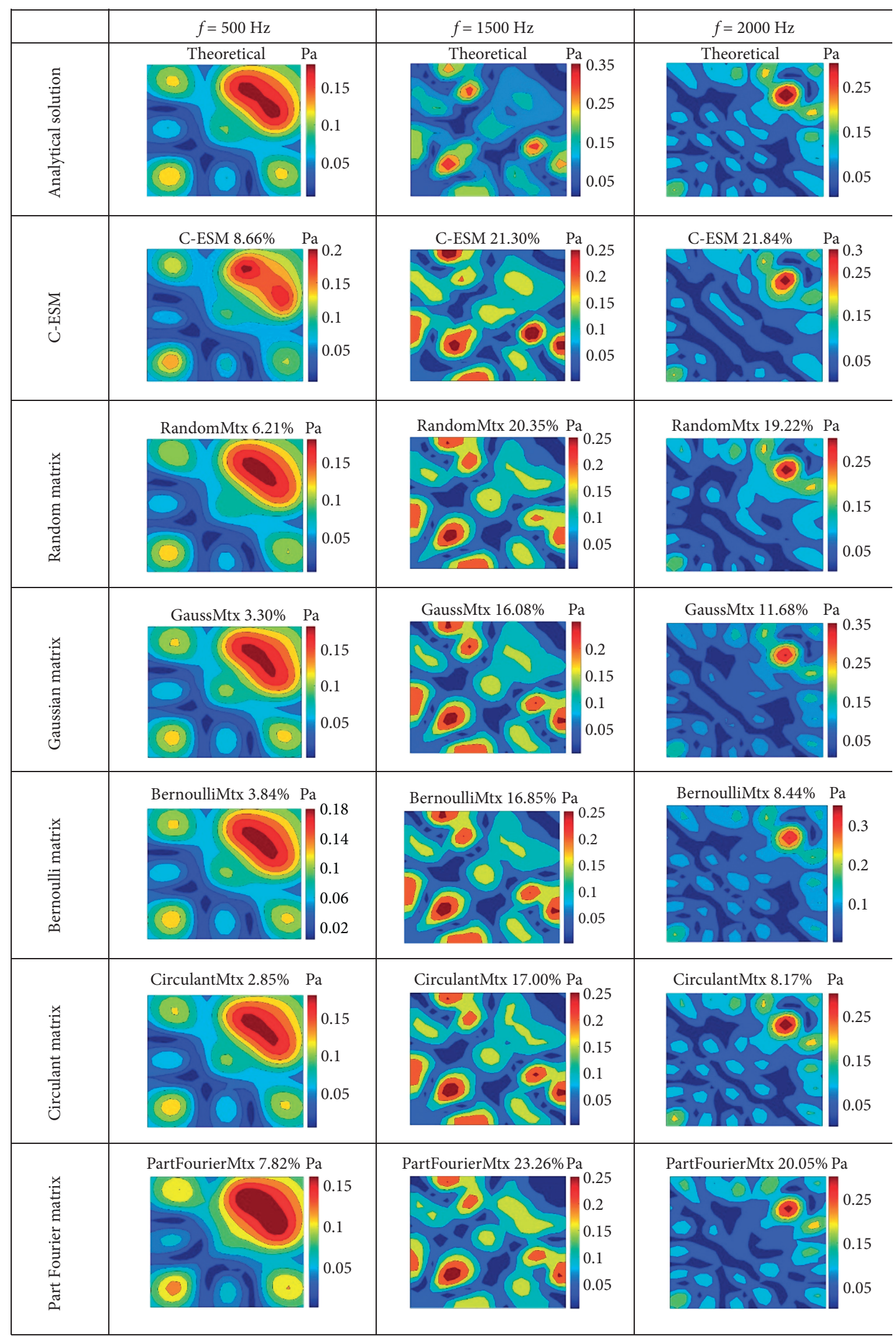

FIGURE 7: Reconstructed and theoretical acoustic pressure nephogram of six methods with 64 sampling points. 


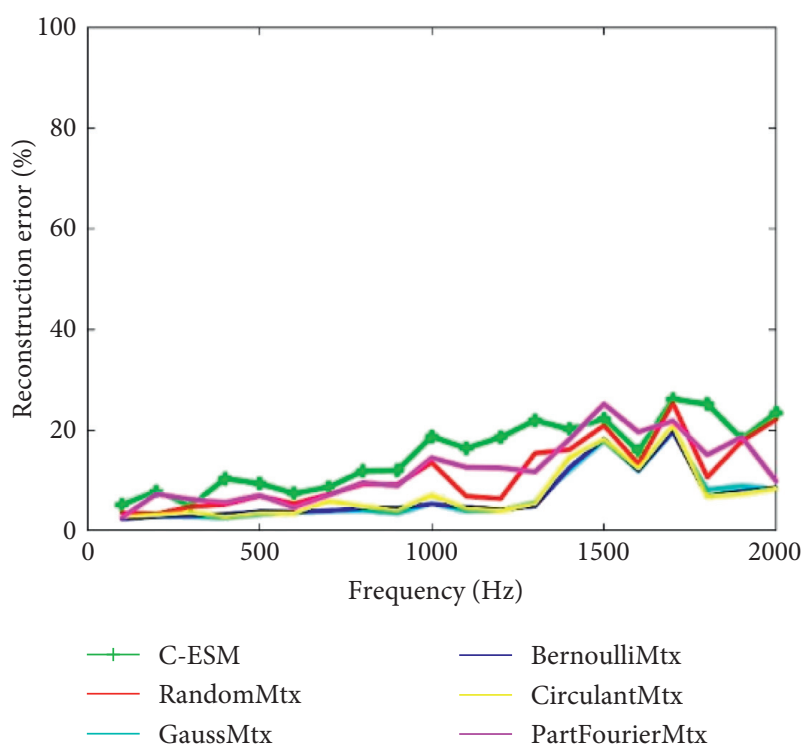

Figure 8: Acoustic pressure reconstruction error with frequency for various acoustic field reconstruction methods with 64 sampling points.

TABLE 1: Comparison of full name and abbreviation in this article.

\begin{tabular}{lc}
\hline Full name & Abbreviation \\
\hline Boundary element method & BEM \\
Basis pursuit de-noising & BPDN \\
Compressive equivalent source method & C-ESM \\
Compressed fused model equivalent source & CFMESM \\
method & \\
Compressed velocity-mode equivalent source & CVMESM \\
method & CS \\
Compressed sensing & DFT \\
Discrete Fourier transform & ESM \\
Equivalent source method & FTGV \\
Fused total generalized variation & NAH \\
Near-field acoustic holography & NP \\
Nondeterministic polynomial & SFT \\
Spatial Fourier transform & SNR \\
Signal-to-noise ratio & WSM \\
Wave superposition method &
\end{tabular}

further studied in other applications of near-field acoustic holography.

\section{Conclusions}

(1) Based on the ESM, the source intensity can be expanded into a bidirectional Fourier series on the rectangular virtual surface, and a semi-analytical and semi-numerical expression of acoustic pressure can be obtained. The proposed method has higher accuracy compared with the traditional numerical discrete source methods. Furthermore, since this method converts the source intensity vector into the sparse Fourier coefficient vector, the vector to be solved also has stronger sparsity and can be more effectively solved using the $l_{1}$ norm.
(2) Correlation in the measurement matrix is reduced by constructing a random sampling matrix, and the sparse sampling matrix is introduced by expanding it into the sampling matrix. The sparse sampling matrix not only includes the randomness of the sampling position, but also includes the randomness of the sum coefficient of the acoustic pressure at the measuring point, which further reduces correlation in the coefficient matrix.

(3) The acoustic field reconstruction results of the traditional C-ESM and the proposed method for a simply supported plate source were compared. The simulation results show that the reconstruction error of the proposed method is lower than that of the C-ESM, and this method has higher accuracy with the Gaussian matrix, Bernoulli matrix, and circular measurement matrix. In particular, the errors of the Gaussian matrix and circular measurement matrix under central excitation are about less than $16 \%$ in the $1400 \sim 3000 \mathrm{~Hz}$ frequency range, which are at least $4 \%$ lower than the C-ESM.

(4) In this study, center excitation and arbitrary eccentric excitation are used for the excitation of a simply supported plate. The sound field reconstruction of a simply supported plate under other arbitrary excitations can be regarded as the superposition of the excitations in this study. Therefore, this method is of great significance for planar sound sources, but for the sound field reconstruction of nonplanar sound sources, especially the sound field reconstruction of rotating structures, this method can be further studied (Table 1).

\section{Data Availability}

The data used to support the findings of this study are available from the corresponding author upon request.

\section{Conflicts of Interest}

The authors declare that they have no conflicts of interest.

\section{Acknowledgments}

The research was supported by the National Natural Science Foundation of China under grant no. 51775121 and Doctor Start-up Fund of Guangxi University of Science and Technology (No. 03210126). The supports are gratefully acknowledged.

\section{References}

[1] E. G. Williams and J. D. Maynard, "Holographic imaging without the wavelength resolution limit," Physical Review Letters, vol. 45, no. 7, pp. 554-557, 1980.

[2] W. A. Veronesi and J. D. Maynard, "Nearfield acoustic holography (NAH) II. Holographic reconstruction algorithms and computer implementation," Journal of the Acoustical Society of America, vol. 81, no. 5, pp. 1307-1322, 1987. 
[3] K. A. Cunefare and G. Koopmann, "A boundary element method for acoustic radiation valid for all wavenumbers," The Journal of the Acoustical Society of America, vol. 85, no. 1, pp. 39-48, 1989.

[4] G. H. Koopmann, L. Song, and J. B. Fahnline, "A method for computing acoustic fields based on the principle of wave superposition," Journal of the Acoustical Society of America, vol. 86, no. 6, pp. 2433-2438, 1989.

[5] T. He, V. F. Humphrey, S. Mo, and E. Fang, "Three-dimensional acoustic scattering from transversely symmetric surface waves in deep and shallow water using the equivalent source method," The Journal of the Acoustical Society of America, vol. 148, no. 1, pp. 73-84, 2020.

[6] G. J. Too and B. H. Wu, "Application of similar source method for noise source identification," Mechanical Systems \& Signal Processing, vol. 21, no. 8, pp. 3167-3181, 2007.

[7] D. L. Donoho, "Compressed sensing," IEEE Transactions on Information Theory, vol. 52, no. 4, pp. 1289-1306, 2006.

[8] M. Elad, Sparse and Redundant Representations from Theory to Applications in Signal and Image Processing, Springer Publishing Company, New York, NY, USA, 2010.

[9] W. L. Chan, K. Charan, D. Takhar, K. F. Kelly, R. G. Baraniuk, and D. M. Mittleman, "A single-pixel terahertz imaging system based on compressed sensing," Applied Physics Letters, vol. 93, no. 12, pp. 101-105, 2008.

[10] G. Chardon, L. Daudet, A. Peillot, F. Ollivier, N. Bertin, and R. Gribonval, "Nearfield acoustic holography using sparsity and compressive sampling principles," The Journal of the Acoustical Society of America, vol. 132, no. 3, pp. 1521-1534, 2012.

[11] G. E. Fernandez and A. Xenaki, "The equivalent source method as a sparse signal reconstruction, J. Inter-noise and Noise-con Congress and Conference proceedings," Institute of Noise Control Engineering, vol. 250, no. 1, pp. 5731-5740, 2015.

[12] G. E. Fernandez, A. Xenaki, and P. Gerstoft, "A sparse equivalent source method for nearfield acoustic holography," Journal of the Acoustical Society of America, vol. 141, no. 1, pp. 532-542, 2017.

[13] D. Y. Hu, H. B. Li, Y. Hu, and Y. Fang, "Sound field reconstruction with sparse sampling and the equivalent source method," Mechanical Systems and Signal Processing, vol. 108, pp. 317-325, 2018.

[14] D. Y. Hu, X. Y. Liu, Y. Xiao, and Y. Fang, "Fast sparse reconstruction of sound field via bayesian compressive sensing," Journal of Vibration and Acoustics, vol. 141, no. 4, pp. 1-9, Article ID 41017, 2019.

[15] Y. He, L. Chen, Z. Xu, and Z. Zhang, "A compressed equivalent source method based on equivalent redundant dictionary for sound field reconstruction," Journal of Applied Sciences, vol. 9, no. 4, p. 808, 2019.

[16] C. X. Bi, Y. Liu, Y. B. Zhang, and L. Xu, "Extension of sound field separation technique based on the equivalent source method in a sparsity framework," Journal of Sound and Vibration, vol. 442, pp. 125-137, 2018.

[17] F. G. Efren and D. Laurent, "Compressive acoustic holography with block-sparse regularization," The Journal of the Acoustical Society of America, vol. 143, no. 6, pp. 3737-3746, 2018.

[18] C. S. Burrus, Fast Fourier Transforms, CRC Press, Florida, FL, USA, 2012.

[19] Y. Xiang, J. Lu, and Y. Y. Huang, "A fast wave superposition spectral method with complex radius vector combined with two-dimensional fast Fourier transform algorithm for acoustic radiation of axisymmetric bodies," Journal of Acoustic and Vibration, vol. 331, no. 6, pp. 1441-1454, 2012.

[20] E. G. Williams, "Fourier acoustics: acoustic radiation and nearfield acoustical holography," Journal of the Acoustical Society of America, vol. 108, no. 4, p. 1373, 1999.

[21] E. V. D. Berg and M. P. Friedlander, "Probing the pareto Frontier for basis pursuit solutions," SIAM Journal on Scientific Computing, vol. 31, no. 2, pp. 890-912, 2008.

[22] R. Baraniuk, "A lecture on compressive sensing," IEEE Signal Processing Magazine, vol. 24, no. 4, pp. 118-121, 2007.

[23] E. Candes, J. Romberg, and T. Tao, "Robust uncertainty principles: exact signal reconstruction from highly incomplete frequency information," IEE Transactions on Information Theory, vol. 52, no. 2, pp. 489-509, 2006.

[24] G. S. Zhang, S. H. Jiao, X. Xu, and L. Wang, "Compressed sensing and reconstruction with Bernouli matrices," in Proceedings of the 2010 IEEE International Conference on Information and Automation, Harbin, China, June 2010.

[25] W. Yin, S. Morgan, J. F. Yang, and Y. Zhang, "Practical compressive sensing with Toepliz and circulant matrices," in Proceedings of the Visual Communications and Image Processing, July 2010.

[26] J. Hald, "A comparison of compressive equivalent source methods for distributed sources," The Journal of the Acoustical Society of America, vol. 147, no. 3, pp. 2211-2221, 2020. 